\title{
Student and Lecturer Partnership: Using visual research to change and address a practice problem
}

\author{
Elizabeth Cooper (University of Chester), Elanor Parks (former undergraduate student \\ nurse, now alumna, University of Chester) Victoria Ridgway (University of Chester)
}

\section{Introduction}

This paper reports upon a research study conducted in partnership between academics and a (then) student, as part of a university internally-funded study. This partnership approach aimed for the active involvement of the student in a pedagogical research project. The student, a current pre-registration nursing student (Elanor) had undertaken a placement with the Joanna Briggs Institute as part of her educational programme and this project enabled her to further her emerging interest in nursing research. Both academics, studying for doctorates and thus beginning their research career, used the award of this project to enhance their research practice. This paper offers a short insight into the journey of the research, from planning to completion and dissemination.

\section{Collaborative research}

Within this study, the principles of participatory research were applied (Wicks, Reason and Bradbury, 2008, with Elanor's student perspective actively sought, listened to and negotiated within the stages of data collection and analysis. Although academics can offer a strategic view of education - which, within nursing, include the inclusion of Nursing and Midwifery Council (NMC) standards (NMC, 2015), Quality Assurance Agency (QAA) standards (2014) and university policies - they are no longer 'living' the experience. This was certainly true of the academics seeking to undertake this study, who had been practising in nurse education for twelve years. Thus, another perspective was needed.

Her first hand contribution constitutes an innovative practice within nurse education research - which has previously taken the 'outsider' stance, with researchers 'looking into' another person's world or problem (Parahoo, 2014) especially at a time when, as has been suggested (Stein et al, 2016), collaborative research in academia is overshadowed by a drive for individualised work. Thus the partnership and collaborative approaches within this study signify an innovative move towards student engagement, which benefits and enhances nurse educational research (Denzin and Lincoln, 2011; Parahoo, 2014) and provides a positive culture of engagement (STARSurg Collaborative, 2017) for peers to embed further student partnerships.

Nurse education research is a crucial component in the improvement and enhancement of student experience and of academic pedagogical practice. In tandem with the focus upon student engagement and collaboration in teaching, learning and assessment as a way of enhancing education (QAA, 2014), student involvement in research offers 'value-added' learning and development opportunities. This project, utilising the educational principles of partnership (QAA, 2014), in which co-production, enhancement and transformational learning occurs, involved a three-way partnership, according with the Higher Education Academy's 'relationship where everybody is actively engaged in...the process of learning and working together' through the completion of a 'real life' research activity (Healey, Flint and Harrington, 2014, page7). The partnership was based upon the concepts of collaboration, equality and an expectation of mutual learning, which are core to the critical educational philosophy underpinning nurse education (Friere, 1970; NMC, 2008). The 
collaborative relationship provided the 'culture' for personal learning, in addition to moving student partnership into a new phase of engagement. Although research has been well used in nurse education to find out what students think, feel or want from their education, students' conducting research is a lesser-known practice. There remain ethical and practical barriers to nursing students' conducting their own research while in practice (Bradnury-Jones and Alcock, 2010), but within the realms of education there is less risk..

Even so, there are limited opportunities for all students to undertake research with academics and thus any sharing of learning from such collaborative research as this may help to compensate, incorporating as it does Elanor's student perspective (insider lens) to enrich and corroborate findings and analysis (Lykes and Mallona, 2008).

\section{The practice problem}

Within the UK, pre-registration nursing students have to attend placements which provide a clinical learning environment NMC, 2010). The learning environments have to meet with NMC standards (NMC, 2013, 2016), verifying that the students are able to acquire their professional nursing competency within the placement. The standards require that the learning environment provides safe, effective nursing care, has adequately-educated nurse mentors and offers learning opportunities appropriate to the practice requirements of the NMC (2010) curriculum. In meeting these criteria, placements within nursing homes, which are usually privately-owned, satisfy the provision of learning opportunities in a non-National Health Service (NHS) context, whilst also offering opportunities for students' to work with a nurse mentor (NMC, 2008) and to learn the essential caring skills (NMC, 2010) that underpin professional nursing practice. Nursing homes thus present a useful alternative placement experience for nursing students, enabling them to meet the learning outcomes of the programme.

The use of nursing homes as placements is not new to nurse education (Kerridge, 2008; Wade and Skinner, 2001); however, there is little UK research available to support the growth of this area as a source of placements.. Furthermore, expansion of placements in nursing homes, as potential positive learning experiences, is inhibited by stereotypical views of them. In the current curricula, the need for students to experience home-based care is required (NMC, 2010), whilst the vision for nurse education (Willis, 2012) anticipates a growing need for students to be competent in delivering care in community environments. Nursing-home placements do offer a practical solution to the increasing demand for suitable community-based learning environments. As a possible answer to the growing problem of ensuring sufficient placements for nursing students, this study sought to ascertain what nursing students thought of nursing-home placements.

Project specifications and research methods

The research aimed to establish what were student nurses' perceptions of nursing-home placements. A mixed qualitative methodological approach was employed: visual methods (drawings) and photo elicitation (focus groups). Visual methodology - and, in particular, drawings - allows the articulation of concepts that participants find difficult to express, therefore capturing hidden emotions and beliefs (Guillemin, 2004). It has been suggested that the use of drawings 'speaks a thousand words' and engenders a deeper understanding of , including social and cultural structures and processes (Pink, 2007; Rose, 2012), as, for example, a clinical learning environment. Drawings have also been found helpful in establishing whether stereotypical views exist (Mead and Metreaux,1957; Samaras, Bonoti and Christidou, 2012). Visual methods have been used successfully to record clinical learning by: Spouse (2003), when student nurses drew images of their experiences during the programme; Devine-Wright et al (2009), who used drawings to generate focus-group 
discussion about participants' understanding of electricity; Guillemin (2004), when exploring women's perceptions of heart disease.

The concept of interviewing within the sphere of visual methods has been advocated by several authors (Margolis and Pauwels, 2011; Pink, 2007; Rose, 2012) and is referred to as 'photo elicitation', which, in simple terms, is when a photograph produced by a participant is used in an interview as a discussion point. Drawings were applied in this instance. These methods were chosen to facilitate students' participation and engage them in a learning style that was familiar (they being visual learners) and to demystify research by turning it into manageable and coherent tasks. This in turn would enable and develop the students' research knowledge.

Ethical approval was gained from the University. Twelve undergraduate nurses who had been allocated to a nursing home for their clinical placement were recruited to gain a collective insider response to a practice problem. A third-year undergraduate student nurse was recruited to the research team to provide a student context to the analysis and discussion of findings.

At the first data collection point, the sample members were asked to draw what they thought a nursing-home placement experience would be like; they then discussed this with others in a recorded focus group (photo elicitation). The second data collection point, following their ten-week clinical experience in a nursing home, repeated this process. The drawings were thematically analysed using Rose's (2012) three-step thematic framework, whilst a basic thematic analysis was applied to the focus groups. The drawings were first analysed by the student participants during the photo elicitation interviews, during which explanations and meanings of their drawings were shared with their peers and images were questioned and challenged. Secondly, the research team explored both the drawings and photo elicitation transcripts.

\section{Opportunity for learning: Focus-group interview}

Completing the focus-group interview provided a useful shared learning between academic and student. The purpose of the focus group, to elicit feelings and thoughts relating to the participants' drawings, required facilitative, group-management and observational skills (Parahoo, 2014). This presented a challenge for Elanor, who had yet to develop groupmanagement skills; the pre-placement focus groups did provide a useful opportunity for her to use 'nursing' observational skills in a new context. Acting as an observer, Elanor complemented the facilitation of the group discussion, adding her own questions and 'fieldnotes' to the observation. After completion of the focus group, the field notes were kept as a way of recording initial thoughts, ideas, themes from the students' discussion. These were utilised within the researchers' collaborative analysis of all the pre-placement data.

Findings:

Two dominant themes were identified across the pre- and post-data collection points: 1) students' perceptions of nursing homes, which focused on placement anxiety, the nursinghome environment and care of the person; 2) student learning.

\section{Perceptions of nursing homes}

The narrative and images obtained from the pre- and post-placement focus groups illustrated a journey of enlightenment for the students. Typically, they dreaded the 
placement, seeing it as an environment in which they would not learn 'nursing skills' and anticipating that nursing care there would be basic and unfulfilling. They also made the point that they felt disadvantaged in comparison to their peers. Words and images used to describe a nursing home included 'prison' and a place where older people lived and went to die. The image below provides a typical example of the students' perceptions.

Pre-placement image reflecting perceptions of nursing homes

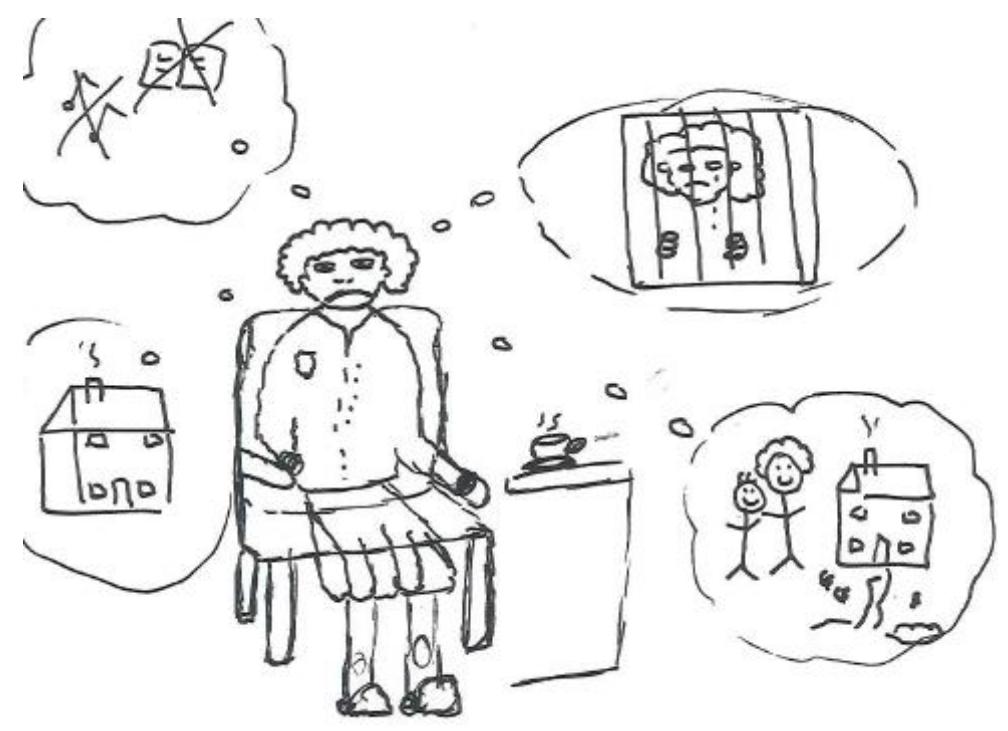

The transition in perceptions was notable in the post-placement focus group: they now appreciated their learning experience and were proud of their achievements. Importantly, the drawings were more positive portrayals of nursing homes and were more individualised, as evidenced in the image below.

Post-placement image, reflecting changed perceptions of nursing homes
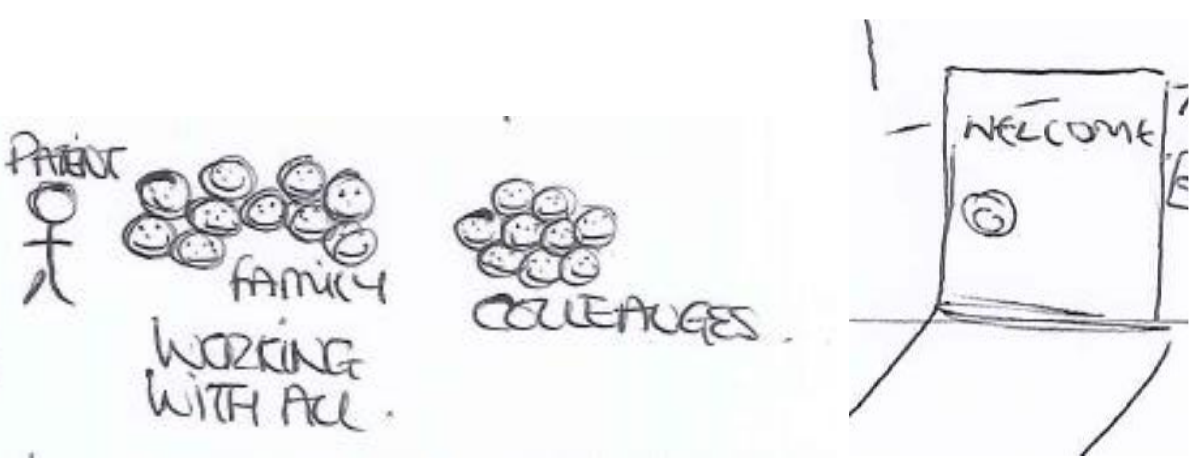

\section{Learning}

Pre-placement learning suggested that the students were concerned about their learning opportunities: for example, they depicted themselves as puzzled and confused or even running away. However, the students changed their view of nursing homes after completion of the placement: they recognised that, although they learnt core 'skills' such as hygiene and communication, there was a growing appreciation of the role of the registered nurse within this care environment. The drawings and subsequent focus groups depicted a positive view of caring for older residents, a valuing of the 'community' feel amongst carers, nurses and 
residents and recognition of the valuable and key role their mentor played in supporting their learning. In tandem with an increased positive feeling, the mentors' role was considered pivotal in ensuring that students were actively learning during their placement.

Pre-placement image reflecting learning
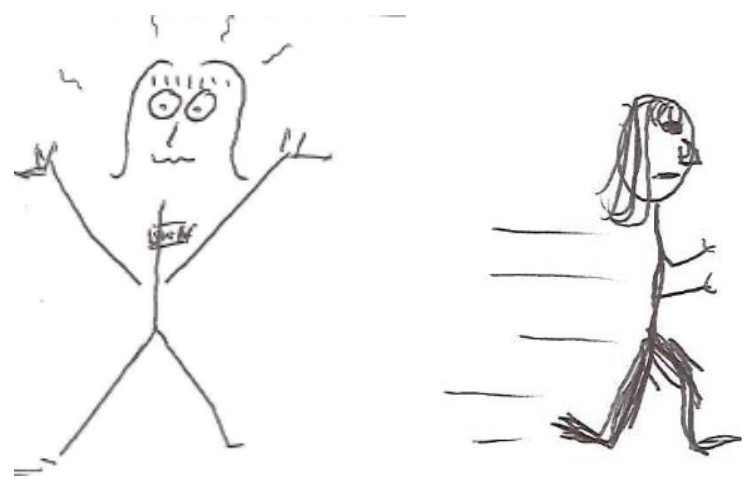

Post-placement image reflecting learning
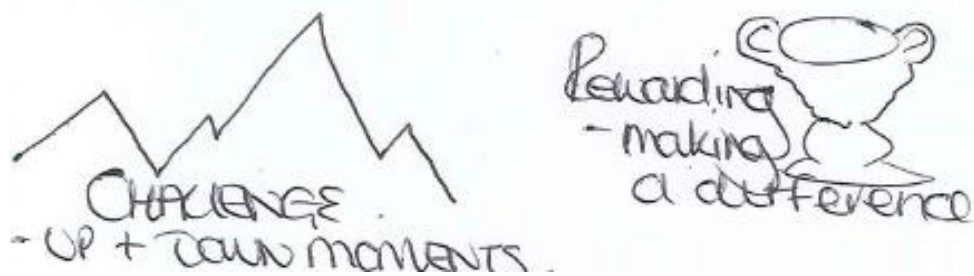

Discussion

The active involvement in the focus groups of the participants and Elanor, the student coresearcher, enabled peer learning, discussion of ideas and sharing of experiences. Through this collaborative relationship, which fostered a culture of mutual learning and understanding, the co-production of themes emerged.

The positive view of the learning environment in the nursing home mirrors previous findings (Abbey, Abbey, Bridges and Elder, 2006; Brynildsen, Bjork, Berntsen and Hestetun, 2014). Students in this study held similar beliefs relating to prior perceptions about limited learning, completion of 'basic' skills and lack of technical skills (Abbey et al, 2006). In fact, post placement, a revised view of nursing homes as communities favourably influenced both the students' attitude to the caring environment and their appreciation of the concept of personcentred care, which, within the UK, is considered the way forward in improving the NHS; the students in this study appeared to grasp precisely why this is the case.

Pleasingly, the students' pictures post placement implied an improved view of aged care, which may have beneficial impact upon the nursing of this client group; such attitude adjustment reflects previous authors' work (Lea et al, 2014). The UK's change in population demographic turns the focus of all nursing services upon caring for the nation's ageing 
population and the attitudinal changes evident in this student group signify a positive outcome for the study.

Instrumental to the changes in students' views and their learning of 'basic' nursing skills was the role of the mentor. This contrasts with Brynildsen et al (2014), whose students scored preceptor support as 'unsatisfactory'. In line with professional guidance and with UK research, the mentor acted as a guide and facilitator, helping students to maximise their inplacement learning. The enthusiasm for supporting nursing students has been attributed to the mutual learning achieved, attributed to the fresh eyes, recent knowledge and questioning behaviours between mentor and student (Grealish, Bail and Ranse, 2010). This is an interesting finding, when there is an increasing imperative to move away from the UK's oneto-one model of nurse mentorship, owing to pressures on and within the National Health Service (Willis, 2012). These findings concur with Kerridge (2008), that, by ensuring that the mentor within the nursing homes is prepared, enthused and supported, nursing students' learning of person-centred care within a home-care environment is achievable. Thus, concerns about the use of nursing homes as placements may be unfounded, as long as mentors remain effective.

Reflection on the Partnership

\section{Dissemination}

The funding of this project enabled the team to present its findings at the British Gerontological Society Conference in 2015. This conference had an audience of practitioners from a range of professional specialisms, including nurse education and health and social care organisations. The findings were generally well received, especially in relation to the potential for placing nursing students in nursing homes, the use of visual methods and, most importantly, the collaboration achieved by enlisting a student as a coresearcher. As the conference continued, delegates' discussions explored the potential benefits - for educators, placement staff and nursing-home residents - of enhanced interaction with students, as well as the mutual learning, promotion of evidence-based practice and the positive influence upon both staff and students, especially when the latter have roles as co-researchers. Thus, within the realms of nursing homes and gerontological care, the presence of nursing students was found to have potentially more benefits than were initially raised in the discussion. Importantly, the presence of our student co-researcher offered the audience valuable personal perspectives; also, significantly, at a time of sharp focus on collaboration with service users, the changes in students' perceptions about nursing homes arising from their experiences were seen as very positive, and our partnership as innovative and unique.

Reflexive piece

As a new collaborative researcher, sharing this experience with my student was a naturally exciting prospective to undertake. As a nurse, working with people is an embedded part of my life, whereas my research education has focused upon this as a solitary journey. Being in the process of my doctorate, the discovery of my ontological beliefs for my thesis has largely been shaped by the strong medical, positivist influence upon nursing research. Undertaking this study has reaffirmed my beliefs in the importance to find ways to involve participants in research, as this helps to give true accounts of their perspectives from the 
data, which, will inform my doctoral thesis and future research practice. Overall, the experience offers new options for student engagement in education.

Student partnership

The collaborative partnership has provided new insights into conducting research with professional colleagues and students. Elanor was an engaged, enthusiastic and equal member of the team. During data collection and analysis, she brought a unique lens and meaning to the data and concepts under investigation. From my own perspective, working in partnership with a 'novice' researcher made me reflect upon and reconsider my own knowledge of research and how I facilitated research projects, explained information and shared the workload. The highlight of the research journey to date was the preparation of an abstract for an international conference and the subsequent presentation of research. Elanor co-prepared the presentation and had an equal part in the presentation at the conference. Interestingly, she was the only undergraduate student presenter. Found by her audience to be engaging, professional and knowledgeable, she was frequently stopped and questioned by other conference participants. We have since presented this research at a further international conference and university staff day and, each time, Elanor's presence and participation added a unique slant to the research. We have gained much from her involvement. What has surprised me through this journey is the paucity of student partnership research outside the 'student engagement/partnership' forums. On a personal note, this has inspired me to continue on this journey of partnership, as I can see the benefits, not only for the research process, but also for the individual student. Elanor has now successfully qualified as a registered nurse and is engaged in postgraduate study and research.

Student partnership: reflection

Elanor took an active role in engaging with the process of the study. She attended the initial focus-group interview and had a vital role in meeting and greeting students and taking field notes. The use of field notes in this instance aided the development of themes from the preplacement data. Following the focus group, we met to review the drawings and Elanor's insight into the feelings they implied, the students' emotional preparation for placements and the meeting with their mentor; this all helped to elicit the initial themes relating to how students anticipated the nursing-home placement. During the post-placement discussions, Elanor's insights as a learner helped to identify the 'learning role' theme, which was highly relevant to the mentor and her/his relationship with the learner and identified the significance of this individual for the student. This supports the literature relating to nursing-home placements and the importance of developing good mentor role models into this area of nursing work.

Reflection on implications for practice

The use of students as partners and co-researchers - and/or their being actively involved has provided an insider view and given us the opportunity to reflect on the implications for future practice. In particular, the following points focus on helping to solve the practice problem and how we engage students and facilitate their role as co-researchers and partners. We suggest that: 
- student participation and collaboration provides a unique insight into the practice problem;

- the use of drawings allows the mundane to be captured and perceptions to become a narrative.

We recommend:

- the selection and consideration of this research method (drawings and photo elicitation) in collaboration with student partner/s.

\section{Conclusion}

Student partnership to identity a problem and deliver change through this project has been successful. As equal partners, we have all gained and learnt from the experience. The lessons learnt in this instance are positive and promote the continued engagement of students as partners and co-researchers. That we experienced no pitfalls in this partnership may be attributed to several factors, including effective communication, equality in decision making and partnership, consideration of each other's own unique perspective, the willingness and motivation of the student partner, thorough preparation of the project and research design and identification of individual researchers' learning needs. To this end, we advocate the engagement of students as co-researchers.

\section{Reference list}

Abbey, J., Abbey, B., Bridges, P. and Elder, R. (2006) 'Clinical Placements in residential care facilities: The impact upon nursing students' perceptions of aged care and the effect on future career plans.' Australian Journal of Advanced Nursing, 23(4), 14-19.

Bradnury-Jones, C. and Alcock, J. (2010) 'Nursing students as research participants: a framework for ethical practice.' Nurse EducationToday, 30, 192-196.

doi:10/1016/j.nedt.2009.07.013

Brynildsen, G., Bjork, I., Berntsen, K. and Hestetun, M. (2014) 'Improving the quality of nursing students' clinical placements in nursing homes: An evaluation study.' Nurse

Education in Practice, 14, 722-728.

Denzin, N. and Lincoln, Y. (2011) The SAGE Handbook of Qualitative Research (4th ed.). Los Angeles, CA: Sage Publications, Inc.

Devine-Wright, H. and Devine-Wright, P. (2009) 'Social Representations of Electricity Network Technologies: exploring process of anchoring and objectification through the use of visual research methods.' British Journal of Social Psychology, 40, 357-373.

Friere, P. (1970) Pedagogy of the Oppressed. London, UK: Penguin Books Ltd.

Grealish, L., Bail, L. and Ranse, K. (2010) 'Investing in the future: residential aged care staff experiences of working with nursing students in a community of practice'. Journal of Clinical Nursing, 19, 2291- 2299. doi: 10.1111/j.1365-2702.2009.03133.x 
Guillemin, M. (2004) 'Understanding Illness: using drawings as a research method.'

Qualitative Health Research,14(2), 279-289.

Healey, M., Flint, A. and Harrington, K. (2014) Engagement through partnership; students as partners in learning and teaching in higher education. Available at:

https://www.heacademy.ac.uk/system/files/resources/engagement through partnership.pdf (Accessed: 12 June 2017.

Kerridge, J. (2008) 'Supporting student nurses on placement in nursing homes.' Nurse Education in Practice, 8(389-396). doi:10.1016/j.nepr.2008.03.003

Lea, E., Marlow, A., Bramble, M., Andrews, S., Crisp, E., Ecclestn, C. . . Robinson, A. (2014) 'Learning opportunities in a residential aged care facility: The role of supported placements for first year nursing students.' Journal of Nurse Education, 53(7), 410-414. Lykes, M. and Mallona, A. (2008) 'Towards Transformational Liberation: Participatory and Action Research and Praxis.' In: Reason, P. and Bradbury, H. (eds.), The SAGE Handbook of Action Research: Participative Inquiry and Practice (2nd ed.). London, UK: SAGE Publications Ltd, 106-120.

Margolis, E. and Pauwels, L. (eds.) (2011) The sage handbook of visual research methods. London: SAGE Publications Ltd.

Mead, M. and Metreaux, R. (1957) 'The image of the scientist among high school students, a pilot study.' Science, 126, 384-390.

Nursing and Midwifery Council (2010) Standards for pre-registration nurse education.

Available at: www.nmc.org.uk. (Accessed: 12 June 2017).

Nursing and Midwifery Council (2008) Standards to support learning and assessment in practice. Available at www.nmc.org/uk. (Accessed: 12 June 2017).

Nursing and Midwifery Council (2013) Quality Assurance Framework. Available at:

www.nmc.org.uk (Accessed: 12 June 2017).

Nursing and Midwifery Council. (2016). Quality Assurance Framework: Available at: www.nmc.org.uk (Accessed: 12 June2017).

QAA. (2014) The UK Quality Code for Higher Education. Available at:

http://www.qaa.ac.uk/publications/information-and-guidance/publication?PublD=181

(Accessed: 12 June 2017).

Parahoo, K. (2014) Nursing research: Principles, process and issues (3rd ed.). Basingstoke, UK: Palgrave Macmillan.

Pink, S. (2007) Doing visual ethnography. London: SAGE Publications Ltd.

Rose, G. (2012) Visual Methodologies, an introduction to researching with visual materials (3rd ed.). London: SAGE Publications Ltd.

Samaras, G., Bonoti, F. and Christidou,V. (2012) 'Exploring Children's Perceptions of Scientists Through Drawings and Interviews.' Social and Behavioural Sciences, 46, 15411546.

Spouse, J. (2002) Professional Learning in Nursing. London: Wiley-Blackwell.

STARSurg Collaborative, (2017) 'Students' participation in collaborative research should be recognised.' International Journal of Surgery. doi:10.1016/j.jisu.2017.01.114

Stein, M.J., Daugherty, A., Rivera, I., Muzzo, J. and Lynn, C.D. (2016) 'Thinking outside anthropology's box: socialising undergraduates through collaborative research, teaching and service.' Annals of Anthropological Practice, 40(2), 164-177. doi: 10.1111/napa.12099 Wade, S. and Skinner, A. (2001) 'Student placements in nursing homes.' Nursing Older People, 13(2), 14-17.

Wicks, P., Reason, P. and Bradbury, H. (2008) 'Living Inquiry: Personal, Political and Philosophical Groundings for Action Research Practice.' In: Reason, P. and Bradbury, H. (eds.), The SAGE Handbook of Action Research: Participative Inquiry and Practice (2nd ed.). London, UK: SAGE Publications Ltd, 15-30.

Willis Commission . (2012) 'Quality with Compassion:the future of nurse education.' Available at: www.nursingtimes.net (Accessed: 12 June 2017). 
